\title{
A simple construction of positive loops of Legendrians
}

\author{
Dishant Pancholi, José Luis Pérez and Francisco Presas
}

\begin{abstract}
We construct positive loops of Legendrian submanifolds in several instances. In particular, we partially recover G. Liu's result stating that any loose Legendrian admits a positive loop, under some mild topological assumptions on the Legendrian. Moreover, we show contractibility of the constructed loops under an extra topological assumption.
\end{abstract}

\section{Introduction}

\subsection{Motivation}

Consider a $(2 n+1)$-dimensional co-oriented contact manifold $(M, \xi)$. Y. Eliashberg and L. Polterovich [EP] introduced the notion of non-negative contact isotopy and showed that it induces a relation on the identity component of the group of contactomorphisms, $\mathcal{C} \mathcal{D}$ if $f_{0}(M, \xi)$. This relation, which was also studied by Bhupal $[\mathrm{Bu}]$, is naturally reflexive and transitive but not necessarily anti-symmetric. We say that $\mathcal{C D}$ if $f_{0}(M, \xi)$ is strongly orderable if the relation is also anti-symmetric, i.e. it is a partial order on $\mathcal{C} \mathcal{D}$ if $f_{0}(M, \xi)$. Analogously, we define a relation in the universal cover $\widehat{\mathcal{C D} \text { if }} f_{0}(M, \xi)$ that again may fail to be anti-symmetric. We say that $\widetilde{\mathcal{C D} \text { iff }} f_{0}(M, \xi)$ is orderable if the relation defines a partial order on $\widehat{\mathcal{C D} i f} f_{0}(M, \xi)$. Contact topology has embraced the study of this relation during the last few years [AFM], [EKP], [EP], [Gi], [We].

There is a relative version of the construction. Let $L$ be a Legendrian submanifold in a contact manifold $(M, \xi)$ and denote by $\mathcal{L} e g(L)$ the space of all Legendrian submanifolds which are Legendrian isotopic to $L$. Non-negative Legendrian isotopies also define a relation on $\mathcal{L} e g(L)$ and we say that $\mathcal{L} e g(L)$ is orderable if this 
relation is anti-symmetric (respectively, the universal cover $\widetilde{\mathcal{L} e g}(L)$ is orderable if the analogous relation is anti-symmetric).

The existence of these partial orders can be checked in terms of the nonexistence of positive loops of contactomorphisms (resp. Legendrians). The study of orderability for Legendrians and of the existence of positive (contractible) loops has been an active research area in contact topology. Thus, for instance, V. Colin, E. Ferrand and P. Pushkar [CFP] studied the non-existence of positive loops of Legendrian submanifolds in the unit contangent bundle $\mathbb{S}\left(T^{*} M\right)$ of a manifold $M$ whose universal cover is the $n$-dimensional real space. In the field of Lorentzian geometry, V. Chernov and S. Nemirovsky [CN1], [CN2], [CN3] apply this topic to the study of causality in globally hyperbolic spacetimes. The orderability property of Legendrians gives rise to the existence of bi-invariant integer-valued metrics on the space of Legendrians [CS].

Recently, G. Liu [Li1], [Li2] has announced the existence of (contractible) positive loops for loose Legendrian submanifolds. The goal of this note is to offer a shorter proof of G. Liu's result under some extra assumptions.

\subsection{Statement of the results}

Consider a $(2 n+1)$-dimensional manifold $M$ endowed with a co-oriented contact structure $\xi$. An $n$-dimensional embedded submanifold $L^{n} \subset M^{2 n+1}$ is called Legendrian if its tangent space at each point is contained in the contact distribution.

Denote by $\mathbb{D}_{\varepsilon}^{n}$ the closed Euclidean ball of radius $\varepsilon$ in $\mathbb{R}^{n}$; and denote by $\mathbb{D}_{\varepsilon}^{n}$ the open ball. The key remark of this article is the following

Theorem 1. Let $(M, \operatorname{ker}(\alpha))$ be a contact manifold and fix an $\varepsilon>0$ positive constant. Consider the contact manifold $\left(M \times \mathbb{D}_{\varepsilon}^{2}(r, \theta), \operatorname{ker}\left(\alpha+r^{2} d \theta\right)\right)$. Any closed Legendrian submanifold in $M \times \stackrel{\mathbb{D}}{\varepsilon}_{\varepsilon}^{2}$ admits a positive loop of Legendrians.

A loop of Legendrian submanifolds is called positive if the generating Hamiltonian of the loop is positive (see definitions below). The proof is extremely simple and the core of this note is devoted to extract some interesting corollaries of the result. The most important one is the next

Theorem 2. Let $n \geq 2$. Fix a loose closed Legendrian submanifold $L^{n}$ in a contact manifold $\left(M^{2 n+1}, \xi\right)$. Assume that its bundle $T^{*} L \oplus \mathbb{R}$ has two pointwise linearly independent sections. Then $L$ admits a positive loop of Legendrians.

Recall that $T^{*} L \oplus \mathbb{R} \simeq J^{1}(L)$ is the normal bundle of the Legendrian submanifold and determines the contact structure on a small neighborhood of the submanifold (Weinstein neighborhood Theorem, see [Ge, Theorem 2.5.8]. A Legendrian 
submanifold is loose if there is a special chart in an open ball intersecting the Legendrian (see Definitions 24, 25). Murphy proved that loose Legendrians satisfy an $h$-principle [Mu]. Note that this definition assumes $2 n+1 \geq 5$.

In 3-dimensional contact topology, there is an analogous older notion [EF]. A Legendrian knot in a contact 3-fold whose complement is overtwisted is called loose. They also satisfy an $h$-principle.

If $2 n+1 \geq 5$, any Legendrian submanifold whose complement is overtwisted is loose. This is a consequence of the parametric and relative nature of the $h$-principle for overtwisted contact structures (see $[\mathrm{BEM}]$ ).

For didactical reasons, we will first prove the following particular case of Theorem 2.

Theorem 3. Let $n \geq 1$. Assume that a closed Legendrian submanifold $L^{n}$ in a contact manifold $\left(M^{2 n+1}, \xi\right)$ satisfies that the bundle $T^{*} L \oplus \mathbb{R}$ has two pointwise linearly independent sections. If $M \backslash L$ is overtwisted, then $L$ admits a positive loop of Legendrians.

We remark that this result covers the 3 -dimensional situation that is not included in Theorem 2.

Observe that the hypothesis of $T^{*} L \oplus \mathbb{R}$ having two independent sections is pretty mild. If $\mathrm{L}$ is orientable, then some sufficient conditions for this hypothesis to be satisfied are:

- $\chi(L)=0$. This, in particular, covers odd dimensional Legendrians.

- $w_{n}(L)=0$. This implies that $w_{n}\left(T^{*} L \oplus \mathbb{R}\right)=0$ and by the definition of this obstruction class in the even dimensional case, the vanishing of the class implies the existence of two independent sections. In particular, this covers even dimensional Legendrians with even Euler characteristic.

- Any Legendrian submanifold whose tangent bundle is trivialized by direct sum with $\mathbb{R}$. This covers all the spheres.

There are simple examples of manifolds not satisfying that property. For instance, $L=\mathbb{C P}^{2}$ is a manifold whose 1 -jet bundle $T^{*} \mathbb{C P}^{2} \oplus \mathbb{R}$ does not admit two independent sections.

Let us move to the study of positive contractible loops. We prove the following key remark.

Theorem 4. Let $(M, \xi=\operatorname{ker}(\alpha))$ be a contact manifold and on the product $M \times$ $\mathbb{D}_{\varepsilon}^{4}\left(r_{1}, \theta_{1}, r_{2}, \theta_{2}\right)$ define the contact form $\tilde{\alpha}=\alpha+r_{1}^{2} d \theta_{1}+r_{2}^{2} d \theta_{2}$. Define the domain

$$
M^{+}=\left\{\left(p, r_{1}, \theta_{1}, r_{2}, \theta_{2}\right) \in M \times \stackrel{\mathbb{D}}{\varepsilon}_{\varepsilon}^{4} \text { such that } 0<r_{1}<r_{2}\right\} .
$$

Any Legendrian embedding $L \hookrightarrow M^{+} \subset M \times \mathbb{D}_{\varepsilon}^{4}$ admits a contractible positive loop of Legendrians on $M \times \mathbb{D}_{\varepsilon}^{4}$. 
This statement implies

Corollary 5. Let $n \geq 3$. Fix a loose closed Legendrian submanifold $L^{n}$ in a contact manifold $\left(M^{2 n+1}, \xi\right)$. Assume that the bundle $T^{*} L \oplus \mathbb{R}$ has four pointwise linearly independent sections. Then, $L$ admits a contractible positive loop of Legendrians.

Again, the hypotheses can be easily checked. They are satisfied, for instance, by Legendrian spheres of dimension $n \geq 3$. Let us consider two more corollaries from Theorem 4.

Corollary 6. If $L \subset\left(\mathbb{R}^{2 n+1}, \xi_{s t d}\right)$ with $n \geq 2$, then $L$ admits a contractible positive loop of Legendrians.

Observe that this statement can be proven using the fact that $\mathbb{S}^{2 n+1}$ admits a contractible positive loop $[\mathrm{EKP}]$, placing $\mathbb{R}^{2 n+1} \subset \mathbb{S}^{2 n+1}$ and making sure that the restrictions of the contact isotopies to the Legendrian submanifold do not cross $\infty \in \mathbb{S}^{2 n+1}$. This can be done by a genericity argument whenever $n \geq 2$. However, the proof presented in this note is more elementary.

Corollary 7. Let $\mathbb{R}^{2 n+1}$ be the Euclidean space equipped with the overtwisted at infinity contact structure $\xi$. If $L \subset\left(\mathbb{R}^{2 n+1}, \xi\right)$ and $n>2$ then $L$ admits a contractible positive loop of Legendrians.

For the precise notion of overtwisted at infinity, see Definition 19.

Acknowledgements. We are grateful to R. Casals, V. Colin, V. Ginzburg, G. Liu and A. del Pino, for useful discussions. The first author is grateful to the Marie Curie Research programme "Indo European collaboration on moduli spaces" that allowed him to visit ICMAT during the development of this project. The first author is also thankful to ICTP, Trieste (Italy), where part of this work was carried out when the author visited there as Simons associate. Second and third authors are supported by the Spanish National Research Projects SEV-2015-0554, MTM2016-79400-P and MTM2015-72876-EXP. Finally, we want to mention the good work of the referee. He has contributed to significantly improve the grammar and mathematical readability of this article.

\section{Preliminaries}

Consider a $(2 n+1)$-dimensional manifold $M$ endowed with a contact structure $\xi$. An embedding of an $n$-dimensional manifold $\phi: L^{n} \hookrightarrow M^{2 n+1}$ is called $L e g$ endrian $\left(^{1}\right)$ if its differential $D \phi: T L \rightarrow \phi^{*}(T M)$ satisfies $D \phi(T L) \subset \xi$.

$\left({ }^{1}\right)$ We will work along the paper with parametrized Legendrians. This is done in order to ease the notation. 
A vector field $X$ on $M$ is called a contact vector field if its flow preserves the contact structure $\xi=\operatorname{ker}(\alpha)$. That is, $L_{X} \alpha=g \alpha$, for some $g \in \mathcal{C}^{\infty}(M)$. A contact form $\alpha$ provides a bijection between the space of contact vector fields and the space of smooth functions as follows. Let $R$ be the Reeb vector field associated to $\alpha$. For any contact vector field $X$, the function $H:=\alpha(X) \in \mathcal{C}^{\infty}(M)$ which satisfies the equations:

(1) $i_{X} \alpha=H$

(2) $i_{X} d \alpha=d H(R) \alpha-d H$

is called the associated Hamiltonian. Conversely, for a function $H \in \mathcal{C}^{\infty}(M)$ there exists a unique contact vector field $X_{H}$ verifying the equations above.

A diffeomorphism $\psi$ of $(M, \xi)$ is a contactomorphism if $\psi_{*}(\xi)=\xi$ or, equivalently, $\psi^{*} \alpha=g \alpha$ for some everywhere positive function $g$ on $M$. An isotopy of contactomorphisms is a smooth diffeotopy $\psi_{t}: M \rightarrow M$ generated by a 1 -parametric family of contact vector fields $X_{t}$, with $t \in[0,1]$. We say that the isotopy is a loop of contactomorphisms if $\psi_{0}=\psi_{1}=I d$.

Let us remark that the above bijection implies that the isotopy is completely characterized by a 1 -parametric family of Hamiltonians $H_{t}: M \rightarrow \mathbb{R}$. Hence, we can make the following definition:

Definition 8. An isotopy of contactomorphisms $\psi_{t}$ is non-negative if its associated family of Hamiltonians $H_{t}$ is non-negative, i.e. $H_{t}(p) \geq 0$ for all $p$ in $M$ and for all $t$ in $[0,1]$. If the inequality is strict, the isotopy is called positive. Analogously we can define positive and non-negative loops of contactomorphisms.

This definition is independent of the choice of contact form $\alpha$ for the given coorientation. Let us point out that when we have a loop of contactomorphisms we can choose the parameter to be defined as $t \in \mathbb{S}^{1}$ i.e. the Hamiltonian can be chosen to satisfy $H_{t}(p)=H_{t+1}(p)$. This is because $H_{t}=\alpha\left(X_{t}\right)$ and clearly $X_{t+1}=X_{t}$. The above definitions can be adapted to this situation.

Definition 9. An isotopy of Legendrian submanifolds is a smooth 1-parametric family $\phi_{t}: L \rightarrow M$ of Legendrian embeddings with $t \in I=[0,1]$. That is, a smooth map $\phi: L \times I \rightarrow M$ such that $\left.\phi\right|_{L \times\{t\}}$ is a Legendrian embedding for all $t$. By a loop of Legendrians based at $\Lambda$ we mean an isotopy of Legendrians such that $\phi_{0}(L)=\phi_{1}(L)=\Lambda$ as submanifolds of $(M, \xi)$.

A basic fact about Legendrian isotopies is the next Legendrian isotopy extension theorem:

Theorem 10. (see, e.g., [Ge, Thm. 2.6.2]) Let $\phi_{t}$ be a given isotopy of a closed Legendrian, then we can extend $\phi_{t}$ by an isotopy $\psi_{t}$ of contactomorphisms satisfying $\psi_{t} \circ \phi_{0}=\phi_{t}$ and $\psi_{0}=I d$. 
We are ready to introduce the concept of positive isotopy of Legendrians.

Definition 11. Let us consider the contact structure $\xi=\operatorname{ker} \alpha$. An isotopy $\phi_{t}$ of Legendrians is called non-negative (resp. positive) if $\alpha\left(\frac{\partial \phi_{t}}{\partial t}(p)\right) \geq 0$ (resp. $\left.\alpha\left(\frac{\partial \phi_{t}}{\partial t}(p)\right)>0\right)$ for all $p \in L$ and for all $t$.

Clearly, these notions are independent of the chosen contact form compatible with the coorientation and of the chosen parametrization. The latter is because for a different parametrization $\tilde{\phi}: L \times I \rightarrow M$, the difference of the vector fields $\frac{\partial \phi_{t}}{\partial t}$ and $\frac{\partial \tilde{\phi}_{t}}{\partial t}$ lies in the tangent space to the Legendrian submanifold $\phi_{t}(L)$ at that point.

According to the previous definition, a loop of Legendrians is called nonnegative (resp. positive) provided the isotopy generating the loop is non-negative (resp. positive). Notice that to have a positive loop of Legendrians is much weaker than to have a positive loop of contactomorphisms. Any extension of a positive Legendrian loop needs be neither a loop of contactomorphisms nor is required to be positive. However, we can easily arrange the extension of a positive (resp. nonnegative) loop of Legendrians to be positive (resp. non negative). This fact will be used afterwards.

Definition 12. A loop of Legendrians $\phi_{t}$ is contractible if there exists a homotopy of loops of Legendrians $\phi_{t, s}$ such that $\phi_{t, 1}=\phi_{t}, \phi_{t, 0}=\phi_{0,1}$ and $\phi_{0, s}=\phi_{0,1}=\phi_{1, s}$.

Remark 13. The existence of a positive loop of a Legendrian $L$ implies that the space $\operatorname{Leg}(L)$ is not orderable. Equivalently, the existence of a contractible positive loop of a Legendrian $L$ implies that the space $\widetilde{\mathcal{L} e g}(L)$ is not orderable.

Define the conjugation of a loop of Legendrian embeddings $\phi_{t}$ by a contactomorphism $\Psi$ as $\Psi \circ \phi_{t}$. It is a new loop of Legendrians based on $\Psi \circ \phi_{0}(L)$. The conjugation of a positive (contractible) loop is clearly a positive (contractible) loop. Since isotopic Legendrians are isotopic through contactomorphisms, we have

Lemma 14. If $L$ admits a positive (contractible) loop of Legendrians through it, then any isotopic Legendrian also admits a positive (contractible) loop of Legendrians through it.

Proof. Let $\varphi_{0}: L \rightarrow(M, \xi)$ be a Legendrian embedding. Assume that there exists $\varphi_{t}: L \times \mathbb{S}^{1} \rightarrow M$ a positive (contractible) loop of Legendrians through $\varphi_{0}$. Moreover, assume that there exists $\phi_{1}: L \rightarrow(M, \xi)$ a Legendrian embedding isotopic to $\varphi_{0}$, i.e. there exists an isotopy of Legendrians $\tilde{\phi}_{t}: L \times[0,1] \rightarrow(M, \xi)$ such that $\tilde{\phi}_{0}=\varphi_{0}$ and $\tilde{\phi}_{1}=\phi_{1}$. By Theorem 10, there exists $\psi_{t}: M \rightarrow M$ a contact isotopy such that $\psi_{t} \circ \tilde{\phi}_{0}=\tilde{\phi}_{t}$. Now, $\psi_{1} \circ \varphi_{t}$ is a positive (contractible) loop of Legendrians embeddings based at $\psi_{1} \circ \varphi_{0}=\psi_{1} \circ \tilde{\phi}_{0}=\tilde{\phi}_{1}=\phi_{1}$. 


\subsection{Formal Legendrians and formal contact structures}

Now denote by $\mathcal{C}$ ont $(M)$ the space of co-oriented contact structures on $M$ and consider the set $\mathcal{D C} \operatorname{cont}(M)=\{(\xi, \alpha, J: \xi \rightarrow \xi)\}$ where $\xi \in \mathcal{C} \operatorname{ont}(M), \alpha$ is an associated contact form and $J$ is an almost-complex structure compatible with $(\xi, d \alpha)$. This set is known as the space of decorated contact structures. Notice that the forgetful map $f: \mathcal{D C} \operatorname{ont}(M) \rightarrow \mathcal{C} \operatorname{ont}(M)$ has contractible fibers. Therefore, it induces a homotopy equivalence and thus it has a homotopy inverse $\iota: \operatorname{Cont}(M) \rightarrow \mathcal{D C} \operatorname{ont}(M)$.

Finally, we define the space of formal contact structures of $M$ as the set of pairs $\mathcal{F C} \operatorname{cont}(M)=\{(\xi, J)\}$, where $\xi$ is a co-oriented distribution of rank $2 n$ on $M$ and $J: \xi \rightarrow \xi$ is an almost-complex structure. Two contact structures $\xi_{1}$ and $\xi_{2}$ are formally equivalent if there exists a family of formal contact structures $\left\{\left(\xi_{t}, J_{t}\right)\right\}$ that connects them. Composing $\iota$ with the forgetful map $\pi: \mathcal{D C}$ ont $\rightarrow \mathcal{F C}$ ont, we get a natural map

$$
j: \operatorname{Cont}(M) \longleftrightarrow \mathcal{F C} \operatorname{Cont}(M) .
$$

There is a natural inclusion $i$ given by:

$$
\begin{aligned}
i: \mathcal{F} \operatorname{Cont}(M) & \longleftrightarrow \mathcal{F} \operatorname{Cont}\left(M \times \mathbb{R}^{2}\right) \\
(\xi, J) & \longmapsto\left(\xi \oplus \mathbb{R}^{2},\left(\begin{array}{l|l}
J & 0 \\
\hline 0 & i
\end{array}\right)\right)
\end{aligned}
$$

Lemma 15. The inclusion map (1) induces an isomorphism

$$
i_{0}: \pi_{0}(\mathcal{F C} \operatorname{ont}(M)) \longrightarrow \pi_{0}\left(\mathcal{F} \operatorname{Cont}\left(M \times \mathbb{R}^{2}\right)\right),
$$

if $M$ is an open manifold.

Proof. Assume $\operatorname{dim} M=2 n-1$. Notice that a formal contact structure on $M$ is a reduction of the structure group to $1 \times U(n-1)$. Hence, considering a formal contact structure on $M$ is equivalent to having a section of the associated bundle $S O(2 n-1) / U(n-1)$. Analogously, having a formal contact structure on $M \times \mathbb{R}^{2}$ is equivalent to choosing a section of the associated $(S O(2 n+1) / U(n))$-bundle.

By [Ge, Lemma 8.1.2], the spaces $S O(2 n-1) / U(n-1)$ and $S O(2 n) / U(n)$ (respectively; $S O(2 n+1) / U(n)$ and $S O(2 n+2) / U(n+1))$ are diffeomorphic. We claim that the homotopy groups $\pi_{k}$ of the spaces $S O(2 n-1) / U(n-1)$ and $S O(2 n+1) /$ $U(n)$ are isomorphic whenever $k<2 n-1$. To check it, we write the long homotopy sequence associated to the fibration $U(n) \rightarrow S O(2 n) \rightarrow S O(2 n) / U(n)$. Now consider the following commutative diagram in which the vertical arrows are the morphisms 
associated to the natural inclusions $U(n) \rightarrow U(n+1)$ and $S O(2 n) \rightarrow S O(2 n+2)$ which induce isomorphisms in the $\pi_{k}$ homotopy groups for $k<2 n-1$ :

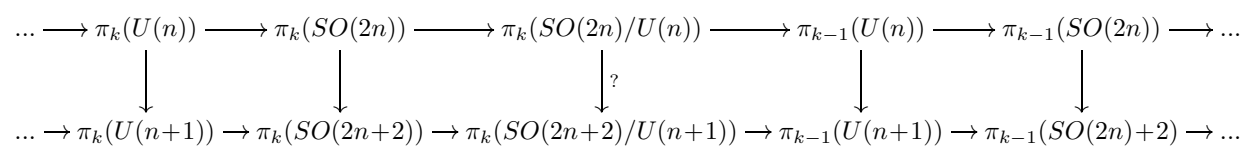

Then, apply the Five Lemma to conclude.

Now obstruction theory shows that $i_{0}$ is an isomorphism if $N$ is open, because $N$ retracts to a $2 n-2$ dimensional skeleton.

Let us remark that if $N$ is closed, the same argument provides the surjectivity of $i_{0}$.

\subsection{Overtwistedness and looseness}

Definition 16. A contact structure $\xi$ on $M^{3}$ is called overtwisted if there exists an embedded 2-disk $\mathbb{D}^{2} \subset M$ such that $\partial \mathbb{D}^{2} \sqcup\{0\}$ is tangent to the contact distribution while the rest of the disk is transverse to $\xi$. If $\xi$ is not overtwisted, it is called tight.

We define the standard overtwisted contact form in $\mathbb{R}^{3}(z, r, \theta)$ to be $\alpha_{o t}=$ $\cos (r) d z+r \cdot \sin (r) d \theta$. It is overtwisted since the embedding $e: \mathbb{D}_{\pi}^{2} \hookrightarrow \mathbb{R}^{3}, e(r, \theta)=$ $(0, r, \theta)$ is overtwisted. For $\delta>0$ small enough, the contact domain $\left(U_{o t}, \alpha_{o t}\right)=$ $\left(\mathbb{D}_{\pi+\delta}^{2} \times[-\delta, \delta], \alpha_{o t}\right)$ always exists in the neighborhood of an overtwisted disk; it will be called the overtwisted contact germ $U_{o t}$.

Overtwisted contact structures are important because they form a subclass of $\mathcal{C}$ ont $(M)$ satisfying a complete $h$-principle [El1], [BEM] in all dimensions. Definition 16 gives us the notion of an overtwisted contact structure in dimension 3 . Let us generalize this concept.

There exists a sequence of positive constants $R(n)$ in $\mathbb{R}^{+}$, whose value is computed in $[\mathrm{CMP}]$ that provide the following

Definition 17. Let $(M, \xi)$ be a contact manifold of dimension $2 n+1>3$. $(M, \xi)$ is called overtwisted if there exists a contact embedding $\phi_{o t}:\left(U_{o t} \times \mathbb{D}_{R(n)}^{2 n-2}, \operatorname{ker}\left(\alpha_{o t}+\right.\right.$ $\left.\lambda_{s t d}\right) \hookrightarrow(M, \xi)$, where $\lambda_{s t d}=\sum x_{i} d y_{i}-y_{i} d x_{i}$ is the standard Liouville form on the closed ball $\mathbb{D}_{R}^{2 n-2}$. The domain will be called the overtwisted contact germ in dimension $2 n+1$.

We say that a formal contact structure is overtwisted if it is genuine in some open set $B$ and is overtwisted in $B$. 
Fix a closed set $A \subset M$ and a contact structure $\xi_{A}$ on a germ of neighborhood of $A$. Denote by $\mathcal{C}_{o n t}\left(M, A, \xi_{A}\right)$ the space of contact structures that are overtwisted in $M \backslash A$ and coincide with $\xi_{A}$ on an arbitrarily small neighborhood $U_{A}$ of $A$. Equivalently, define $\mathcal{F} \operatorname{Cont}_{o t}\left(M, A, \xi_{A}\right)$ to be the space of overtwisted formal contact structures that agree with $\xi_{A}$ on $U_{A}$. Finally, denote by $j$ the inclusion $\operatorname{map} j: \mathcal{C}_{\text {ont }}{ }_{\text {ot }}\left(M, A, \xi_{A}\right) \rightarrow \mathcal{F} \mathcal{C}_{\text {ont }}{ }_{\text {ot }}\left(M, A, \xi_{A}\right)$.

Theorem 18. ([BEM, Thm. 1.2]) If $M \backslash A$ is connected, then the inclusion map $j$ induces an isomorphism

$$
j_{0}: \pi_{0}\left(\mathcal{C}_{\text {ont }} \text { ot }\left(M, A, \xi_{A}\right)\right) \longrightarrow \pi_{0}\left(\mathcal{F C} \text { ont }{ }_{\text {ot }}\left(M, A, \xi_{A}\right)\right) .
$$

In particular, on any closed manifold $M$, any formal contact structure is homotopic to an overtwisted contact structure which is unique up to isotopy.

For the open case, we have

Definition 19. The contact manifold $(M, \xi)$ is called overtwisted at infinity if, for any compact subset $K \subset M$, each noncompact connected component of the contact manifold $(M \backslash K, \xi)$ is overtwisted.

Eliashberg [El2] proved that any two contact structures on $\mathbb{R}^{3}$ overtwisted at infinity are contactomorphic. This result can be extended, without changes in the argument, to general open manifolds of arbitrary dimension. Concretely,

Lemma 20. Let $M$ be an open manifold and let $\left(M, \xi_{0}\right)$ and $\left(M, \xi_{1}\right)$ be two contact structures overtwisted at infinity such that $\xi_{0}$ and $\xi_{1}$ are formally equivalent. Then, there exists a diffeomorphism $\Psi: M \rightarrow M$ such that $\Psi_{*} \xi_{0}=\xi_{1}$.

The proof is left to the reader. It follows, verbatim, [El2].

We claim

Lemma 21. The overtwisted contact germ $\left.\left(U_{o t} \times \mathbb{D}_{R(n)}^{2 n-2}\right), \alpha_{o t}+\lambda_{\text {std }}\right)$ contains an open ball $B_{\text {ot }}$ overtwisted at infinity.

Proof. In 3 dimension, the ball $B_{\text {ot }}$ can be chosen to be the interior of the whole domain $U_{o t}$. The reason is that the contact flow $\partial_{z}$ pushes the overtwisted disk $\mathbb{D}_{\pi}^{2} \times\{0\} \subset U_{\text {ot }}$ arbitrarily close to the boundary.

In higher dimension, the open manifold $C_{o t}=\left(\mathbb{R}^{3} \times \mathbb{R}^{2 n-2}, \alpha_{o t}+\lambda_{\text {std }}\right)$ is overtwisted, because it contains the overtwisted contact germ. Moreover, it is overtwisted at infinity, since $\partial_{z}$ is again a contact vector field that pushes any overtwisted contact germ to infinity. Moreover, $C_{o t}$ admits a formal contact embedding into $U_{o t} \times B_{R}^{2 n-2}(n)$ since both domains are contractible and there is not topological obstruction to upgrade a smooth embedding, that of course exists, into a contact formal one. Then [BEM, Corollary 1.4], changes the formal embedding into a contact one. 
To prove Theorem 3, we will use the above Lemma together with Theorem 1. Hence we will need to find a contact manifold $N$ such that $N \times \mathbb{D}^{2}$ is overtwisted at infinity. The next folklore result shows that it suffices for $N$ to be an overtwisted manifold.

Proposition 22. Let $(N, \operatorname{ker}(\alpha))$ be an overtwisted contact manifold. Then $\left(N \times \mathbb{R}^{2}, \operatorname{ker}\left(\alpha+r^{2} d \theta\right)\right)$ is overtwisted at infinity.

Notice that the proposition does not hold if the dimension of $N$ is 1 since there is no notion of overtwistedness in this case. We need the following elementary

Lemma 23. Let $(M, \xi=\operatorname{ker}(\alpha))$ be a contact manifold satisfying that the Reeb vector field $R_{\alpha}$ is complete. Denote the associated flow $\phi_{t}^{R}$. Choose $f: \mathbb{D}_{r}^{2} \rightarrow \mathbb{R}$ a smooth function and $\lambda \in \Omega^{1}\left(\mathbb{R}^{2}\right)$ a primitive for $\omega_{0}=d x \wedge d y$. Define on $M \times \mathbb{D}_{r}^{2}$ the contact forms $\alpha_{0}=\alpha+\lambda$ and $\alpha_{1}=\alpha+\lambda+d f$. Then the diffeomorphism

$$
\begin{aligned}
\Psi: M \times \mathbb{D}_{r}^{2} & \longrightarrow M \times \mathbb{D}_{r}^{2} \\
(p, x, y) & \longmapsto\left(\phi_{f(x, y)}^{R}(p), x, y\right)
\end{aligned}
$$

satisfies $\Psi^{*} \alpha_{0}=\alpha_{1}$.

The proof is left to the reader.

Proof of Proposition 22. Observe that there exists a smooth function $g: N \rightarrow \mathbb{R}$ such that $\widetilde{\alpha}=e^{g} \alpha$ satisfies that $R_{\widetilde{\alpha}}$ is complete $\left({ }^{2}\right)$. In fact, we have the following diffeomorphism

$$
\begin{aligned}
\psi: N \times \mathbb{R}^{2} & \rightarrow N \times \mathbb{R}^{2} \\
(p, r, \theta) & \mapsto\left(p, e^{g / 2} r, \theta\right),
\end{aligned}
$$

that clearly satisfies $\psi^{*}\left(\widetilde{\alpha}+r^{2} d \theta\right)=e^{g}\left(\alpha+r^{2} d \theta\right)$. So $\left(N \times \mathbb{R}^{2}, \alpha+r^{2} d \theta\right)$ is contactomorphic to $\left(N \times \mathbb{R}^{2}, \widetilde{\alpha}+r^{2} d \theta\right)$. Therefore, we can assume without loss of generality that the Reeb vector field associated to $\alpha$ is complete to begin with.

It is sufficient to show that for any $K>0$, the manifold $W=\left(N \times \mathbb{R}^{2}\right) \backslash(N \times$ $\left.\mathbb{D}_{K}^{2}(0,0)\right)$ is overtwisted. Let us prove it.

First, we realize that since $(N, \operatorname{ker}(\alpha))$ is overtwisted, there exists a positive constant $R=R(n)$ such that $\left(N \times \mathbb{D}_{R}^{2}((0,0)), \operatorname{ker}\left(\alpha+\lambda_{\text {std }}\right)\right)$ is overtwisted [CMP, Thm. 3.1]. Now, let us consider the manifold $\left(N \times \mathbb{D}_{R}^{2}((0, K+3 R)), \operatorname{ker}\left(\alpha+\lambda_{s t d}\right)\right)$ embedded in $W$. We apply Lemma 23 with $f(x, y)=-(k+3 R) x$ to show that $\left.(N \times) \mathbb{D}_{R}^{2}, \operatorname{ker}\left(\alpha+\lambda_{s t d}\right)\right)$ is contactomorphic to $\left(N \times \mathbb{D}_{R}^{2}((0, K+3 R)), \operatorname{ker}\left(\alpha+\lambda_{s t d}\right)\right)$. Hence, $\left(N \times \mathbb{D}_{R}^{2}((0, K+3 R)), \operatorname{ker}\left(\alpha+\lambda_{\text {std }}\right)\right)$ is overtwisted and thus, $N \times \mathbb{R}^{2}$ is overtwisted at infinity.

$\left({ }^{2}\right)$ This is a standard fact: choose $g$ to be a "reasonable" rapidly increasing proper function. 


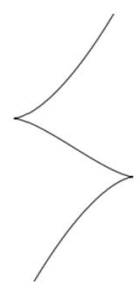

Figure 1. The front projection of a stabilized Legendrian arc.

Just as overtwisted contact structures abide by an h-principle, there also exists a subclass of Legendrian embeddings, referred to as loose, which satisfies an $h-$ principle type result [Mu]. Let us define this class.

A formal Legendrian submanifold $L$ of $M$ is an embedding $\phi: L \rightarrow M$ together with a family $\Phi_{t}: T L \rightarrow \phi^{*} T M$ such that $\Phi_{t}$ is a monomorphism for all $t \in[0,1]$ satisfying that $\Phi_{0}=d \phi$ and $\Phi_{1}(T L) \subset \phi^{*} \xi$. Notice that a Legendrian submanifold can be thought of as a formal Legendrian submanifold by letting $\Phi_{s}=d \phi$ for all $s$. In particular, two Legendrian embeddings $\phi_{0}$ and $\phi_{1}$ are formally isotopic if there exists a smooth isotopy $\phi_{t}$ between them and a homotopy of monomorphisms $\Phi_{t, s}: T L \rightarrow \phi_{t}^{*} T M$ such that $\Phi_{t, 0}=d \phi_{t}, \Phi_{0, s}=d \phi_{0}, \Phi_{1, s}=d \phi_{1}$ and $\Phi_{t, 1}(T L) \subset \Phi_{t}^{*} \xi$.

E. Murphy $[\mathrm{Mu}]$ introduced the notion of loose Legendrian submanifolds. They are characterized by the following local model:

Consider an open ball $\mathbb{D}$ around the origin in $\left(\mathbb{R}^{3}, \xi_{\text {std }}\right)$ where $\xi_{\text {std }}=\operatorname{ker} \alpha_{\text {std }}$ is the standard contact structure on $\mathbb{R}^{3}$ and let $L_{0} \subset\left(\mathbb{R}^{3}, \xi_{\text {std }}\right)$ be a stabilized Legendrian arc as seen in Figure 1. Consider the zero section $\Gamma \subset T^{*} M$ of a closed manifold $M$ and denote by $U_{\Gamma} \subset T^{*} M$ an open neighborhood of it. Then, $\left(L_{0} \times \Gamma \subset\right.$ $\left(\mathbb{D} \times U_{\Gamma}, \operatorname{ker}\left(\alpha_{s t d}+\lambda_{s t d}\right)\right)$ is a Legendrian submanifold.

Definition 24. The pair $\left(L_{0} \times \Gamma, \mathbb{D} \times U_{\Gamma}\right)$ together with the contact structure $\operatorname{ker}\left(\alpha_{s t d}+\lambda_{s t d}\right)$ is known as a loose chart.

Definition 25. A Legendrian submanifold $L^{n} \subset\left(M^{2 n+1}, \xi\right)$ with $n \geq 2$ is called loose if there exists an open set $U \subset M$ such that $((U \cap L, U), \xi)$ is contactomorphic to a loose chart.

The corresponding $h$-principle can be stated as follows.

Theorem 26. ([Mu]) Let $L^{n} \subset M^{2 n+1}$ be a formal Legendrian submanifold with $n>1$. Then, there exists a loose Legendrian submanifold $\tilde{L}$ such that they are formally isotopic. Moreover, given two formally isotopic loose Legendrians $L_{1}$ and $L_{2}$, they are isotopic through loose Legendrians. 


\section{Proof of Theorem 1}

Before proving Theorem 1, we need to introduce a result due to Y. Eliashberg and L. Polterovich [EP] adapted to the Legendrian case by V. Chernov and S. Nemirovski [CN3] which states that if a Legendrian isotopy class contains a nonconstant non-negative loop of Legendrians, then it contains a positive loop. More precisely,

Lemma 27. ([CN3, Prop. 4.5]) Let $\left\{\phi_{t}\right\}$ be a non-negative non-trivial Legendrian loop of closed Legendrians based at L. Then, there exists a positive loop of Legendrians $\left\{\phi_{t}^{\prime}\right\}$ which satisfies that $\phi_{0}(L)=\phi_{0}^{\prime}(L)$.

If we assume that $\phi_{t}$ is contractible then $\phi_{t}^{\prime}$ can be chosen to be contractible.

Theorem 1 is a consequence of the above Lemma.

Proof of Theorem 1. The contact vector field $X=\frac{\partial}{\partial \theta}$ generates a non-negative loop of contactomorphisms, that is positive away from $M \times\{0\}$.

Since $L$ is a Legendrian submanifold in $M \times \mathbb{D}^{2}$ for dimensional reasons there exists a point of $L$ which is not in the contact submanifold $M \times\{0\}$. Hence the loop restricted to the Legendrian is a non-negative non-trivial loop of Legendrians. Now we can apply Lemma 27 to complete the proof.

Corollary 28. Any Legendrian submanifold in $\mathbb{R}^{2 n+1}$ admits a positive loop of Legendrians.

Proof. The standard contact manifold $\mathbb{R}^{2 n+1}$ is nothing but $\mathbb{R}^{2 n-1} \times \mathbb{R}^{2}$ with the contact structure given by $\alpha_{s t d}+r^{2} d \theta$ where $\alpha_{s t d}$ is the standard contact form on $\mathbb{R}^{2 n-1}$. Let $L$ be a closed Legendrian in $\mathbb{R}^{2 n+1}$, by compactness $L \subset \mathbb{R}^{2 n-1} \times \mathbb{D}_{\varepsilon}^{2}$, for $\varepsilon>0$ large enough. The corollary follows from Theorem 1 applied to $\mathbb{R}^{2 n-1} \times \dot{\mathbb{D}}_{\varepsilon}^{2}$.

Remark 29. Actually, it can be shown that $\mathbb{R}^{2 n+1}$ admits a positive loop of contactomorphisms. This is even true for $M \times \mathbb{R}^{2}$ just by checking that the proof of non-negative loop implies positive loops works also for open manifolds [EP, Proposition 2.1.B]. The only delicate issue is that the contact vector fields defined in that proof should be complete.

\section{Proof of Theorem 3}

The main idea of the proof is to construct a neighborhood $U_{L}$ of $L$ contactomorphic to $N \times \mathbb{R}^{2}$ satisfying the hypothesis of Lemma 20. Then the result will follow from Theorem 1. This is the content of Lemma 30. 


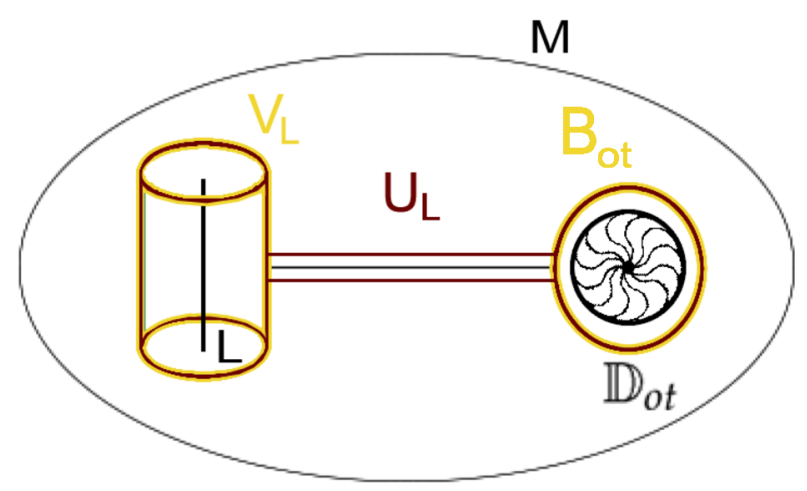

Figure 2. Construction of $U_{L}$.

Lemma 30. For any Legendrian submanifold $L \subset(M, \xi)$ satisfying the hypothesis of Theorem 3 , there exists a neighborhood $U_{L}$ of $L$ diffeomorphic to $N \times \mathbb{R}^{2}$ such that $\left(U_{L}, \xi\right)$ is overtwisted at infinity and $N$ is an open manifold if $n \geq 2$.

Proof. By the first hypothesis and the Legendrian neighborhood theorem [Ge, Theorem 2.5.8], a small tubular neighborhood $V_{L}$ of $L$ is diffeomorphic to $N \times \mathbb{R}^{2}$. By the second hypothesis, there exists an overtwisted disk contact germ which does not intersect $L$. The germ contains an open ball overtwisted at infinity $B_{o t}$ by Lemma 21. $V_{L}$ is disjoint from the overtwisted ball $B_{o t}$. Define $U_{L}$ to be the embedded connected sum of $V_{L}$ with $B_{o t}$ along a tubular neighborhood of a path connecting their boundaries (see Figure 2). $U_{L}$ is overtwisted at infinity by construction and is diffeomorphic to $N \times \mathbb{R}^{2}$.

We want to apply Lemma 20 and we use Proposition 22 to find an overtwisted at infinity contact manifold of type $N \times \mathbb{R}^{2}$. Hence, we have to distinguish two cases.

\subsection{Proof of Theorem 3 for $n>1$.}

It follows from Lemma 30 that there exists a diffeomorphism $\Phi: U_{L} \rightarrow N \times \mathbb{R}^{2}$. In addition, $\left(U_{L}, \xi\right)$ is overtwisted at infinity. By Lemma 15 the submanifold $N \times\{0\}$ can be equipped with a formal contact structure $\left(\xi_{N}, J_{N}\right)$ such that $\left(\xi_{N} \oplus \mathbb{R}^{2}, J_{N} \oplus i\right)$ represents the same formal contact class as $\xi$.

By Theorem 18 there exists an overtwisted contact structure $\xi_{o t}=\operatorname{ker}\left(\alpha_{o t}\right)$ on $N$, formally homotopic to $\xi_{N}$. Therefore, the contact structure $\xi^{\prime}=\operatorname{ker}\left(\alpha_{o t}+r^{2} d \theta\right)$ in $N \times \mathbb{R}^{2}$ is overtwisted at infinity by Proposition 22 and formally homotopic to $\xi$. By Lemma 20, there is a diffeomorphism $F: U_{L} \rightarrow N \times \mathbb{R}^{2}$ taking $\xi$ to $\xi^{\prime}$ and 
preserving co-orientations. By the compactness of $L$, we have that $F(L) \subset N \times \mathbb{D}_{\varepsilon}^{2}$, for $\varepsilon>0$ large enough.

By Theorem 1, $F(L)$ admits a positive loop of Legendrians $\phi_{t}$. Thus, the family $\phi_{t} \circ F^{-1}$ is a positive loop of Legendrians for $L$.

\subsection{Proof of Theorem 3 for $n=1$.}

Let $L \hookrightarrow(M, \xi)$ denote the Legendrian embedding. A tubular neighborhood $U_{L}$ can be identified with $L \times \stackrel{\circ}{D}_{\varepsilon}^{2} \subset(M, \xi)$. By Lemma $30 U_{L}$ is overtwisted at infinity and diffeomorphic to $\mathbb{S}^{1} \times \mathbb{R}^{2}$.

Consider now the contact manifold $\left(\mathbb{S}^{1}(z) \times \mathbb{D}_{\varepsilon}^{2}(r, \theta), \eta=\operatorname{ker}\left(d z+r^{2} d \theta\right)\right)$. Here, integrating $\partial_{z}$ gives a positive loop of contactomorphism with Hamiltonian $H=1$, in particular it is autonomous. Fix a sequence of transverse knots $\gamma_{k}=(z, \varepsilon(1-1 / k), 0)$, with $k \in \mathbb{Z}_{>0}$. Then, the contact manifold obtained as a sequence of half Lutz twists along each of them is overtwisted at infinity. It admits a positive loop of contactomorphisms by $[\mathrm{CP}]$. Denote it by $\left(\mathbb{S}^{1} \times \mathbb{D}_{\varepsilon}^{2}, \eta^{\gamma}\right)$.

Finally, $\xi$ and $\eta^{\gamma}$ are formally equivalent because there exists only one class of formal contact structures on $\mathbb{S}^{1} \times \mathbb{D}_{\varepsilon}^{2}$. Again, the claim follows by using Lemma 20 .

\section{Proof of Theorem 2}

We will use again Theorem 1. Hence we need to construct a neighborhood of $L$ contactomorphic to $\left(N \times \stackrel{\circ}{D}_{\varepsilon}^{2}, \operatorname{ker}\left(\alpha_{N}+r^{2} d \theta\right)\right)$ for some contact manifold $\left(N, \alpha_{N}\right)$.

We first prove a simple case.

\subsection{Euler characteristic zero}

Assume that $T^{*} L$ has a never-vanishing section. Using Weinstein's tubular neighborhood theorem, we find a neighborhood $U_{L}$ of $(L, \operatorname{ker}(\alpha))$ contactomorphic to $\left(T^{*} L \times \mathbb{R}(z), \operatorname{ker}\left(d z-\lambda_{\text {std }}\right)\right)$. As $\left(T^{*} L \backslash\{0\}, d \lambda_{\text {std }}\right)$ and $\left(\mathbb{S}\left(T^{*} L\right) \times \mathbb{R}, d\left(e^{t} \lambda_{\text {std }}\right)\right)$ admit a diffeomorphism preserving the Liouville forms, the natural inclusion $\mathbb{S}\left(T^{*} L\right)$ $\rightarrow T^{*} L \times \mathbb{R}$ is a contact embedding. By the tubular neighborhood theorem for contact submanifolds ([Ge]), there exists a neighborhood $V$ of $\mathbb{S}\left(T^{*} L\right)$ contactomorphic to $\mathbb{S}\left(T^{*} L\right) \times \mathbb{D}_{\varepsilon}^{2}$.

The never-vanishing section of $T^{*} L$ provides an embedding $\sigma: L \rightarrow \mathbb{S}\left(T^{*} L\right) \subset$ $T^{*} L \times \mathbb{R}$. Thus, we obtain a family of embeddings $\sigma_{t}: L \rightarrow T^{*} L \times \mathbb{R}$ defined as $\sigma_{t}=t \sigma$. Since $\sigma_{0}$ is a Legendrian embedding, the whole family $\sigma_{t}$ can be lifted into a family $\left(\sigma_{t}, \Phi_{t}\right)$ of formal Legendrian embeddings. 
Apply Theorem 26 to $\left(\sigma_{1}, \Phi_{1}\right)$ as a formal Legendrian embedding into the manifold to create a family $\left(\sigma_{t}, \Phi_{t}\right)$ with $t \in[1,2]$ of formal Legendrian embeddings in $V$ satisfying that $\sigma_{2}$ is a loose Legendrian embedding. The family $\left(\sigma_{t}, \Phi_{t}\right)$ with $t \in[0,2]$ satisfies the hypothesis of the second part of Theorem 26 and so, it can be deformed relative to $t=0,2$ into a Legendrian isotopy inside $M$. We are, because of Lemma 14, reduced to find a positive loop for the loose Legendrian $\sigma_{2}$. But this is true by Theorem 1 applied to $V$.

\subsection{General case}

By hypothesis, we have that a neighborhood $U_{L}$ of $L$ is diffeomorphic to $N \times \mathbb{R}^{2}$, for an open manifold $N$. By Lemma 15, we assume that there is a formal contact structure $\left(\xi_{N}, J_{N}\right)$ on $N$ such that $\left(\xi_{N} \oplus \mathbb{R}^{2}, J_{N} \oplus i\right)$ is the formal contact class of $\xi$. By Theorem [EM, Thm. 10.3.2], the formal contact structure $\xi_{N}=\operatorname{ker} \alpha_{N}$ can be assumed to be contact.

We are in the hypothesis of [EM, Thm. 12.3.1]. Therefore, the formal contact embedding $e_{0}: N \hookrightarrow N \times\{0\} \subset N \times \mathbb{R}^{2} \simeq U_{L}$ admits an isotopy of formal contact embeddings $e_{t}: N \rightarrow U_{L}$ satisfying that $e_{1}$ is a contact embedding. By the contact neighborhood theorem ([Ge], Theorem 2.5.15), there exists $\phi_{1}: N \times \mathbb{D}_{\varepsilon}^{2} \hookrightarrow U_{L}$, for sufficiently small $\varepsilon>0$, such that

(1) $\left(\phi_{1}\right)_{\mid N \times 0}=e_{1}$.

(2) Fix the contact form $\alpha=\alpha_{N}+r^{2} d \theta$ in the manifold $N \times \stackrel{D}{\mathbb{D}}_{\varepsilon}^{2}$. The map $\phi_{1}$ is a contact embedding.

By construction we have $L \subset N$. Define the family of embeddings $\varphi_{t}: L \rightarrow U_{L}, t \in$ $[0,1]$ as $\varphi_{t}=\left(e_{t}\right)_{\mid L}$.

Promote the family $\varphi_{t}$ into a family of formal Legendrian embeddings $\left(\varphi_{t}, \Phi_{t}\right)$, $t \in[0,1]$. Apply Theorem 26 to $\left(\varphi_{1}, \Phi_{1}\right)$ as formal Legendrian embedding of the manifold $\phi_{1}\left(N \times \mathbb{D}_{\varepsilon}^{2}\right)$, to create a family of formal Legendrians embeddings $\left(\varphi_{t}, \Phi_{t}\right) t \in$ $[1,2]$ such that $\left(\varphi_{2}, \Phi_{2}\right)$ is a loose Legendrian embedding into $\phi_{1}\left(N \times \dot{\mathbb{D}}_{\varepsilon}^{2}\right)$. Since, by hypothesis $\varphi_{0}$ is loose, we can apply the second part of Theorem 26 to show that $\varphi_{0}$ and $\varphi_{2}$ are Legendrian isotopic in $M$.

But the image of $\varphi_{2}$ lies in $\phi_{1}\left(N \times \mathbb{D}_{\varepsilon}^{2}\right)$. Thus, $\left(\phi_{1}\right)^{-1} \circ \varphi_{2}$ is a Legendrian embedding into $\left(N \times \mathbb{D}_{\varepsilon}^{2}, \operatorname{ker}\left(\alpha_{N}+r^{2} d \theta\right)\right)$. Theorem 1 concludes that $\varphi_{2}$ posseses a positive loop. Lemma 14 provides one for the original Legendrian embedding $\varphi_{0}$.

\section{Proof of Theorem 4 and Corollaries}

Proof of Theorem 4. Notice that U(2) acts by contactomorphisms on $M \times \mathbb{D}_{\varepsilon}^{4}$. 
Now consider the contact vector fields $X_{1}=\partial_{\theta_{1}}$ and $X_{2}=\partial_{\theta_{2}}$ with associated Hamiltonians $H_{1}=r_{1}^{2}$ and $H_{2}=r_{2}^{2}$, respectively. The contact vector field $X=X_{2}-$ $X_{1}=\partial_{\theta_{2}}-\partial_{\theta_{1}}$, whose associated Hamiltonian is $H=r_{2}^{2}-r_{1}^{2}$, generates a loop that preserves $M^{+}$and is positive on this domain. Denote by $A_{t}$ the unitary matrix

$$
\left(\begin{array}{cc}
e^{2 \pi i t} & 0 \\
0 & e^{-2 \pi i t}
\end{array}\right)
$$

then the flow associated to $X$ reads as $\phi_{t}\left(p,\left(\begin{array}{c}z_{1} \\ z_{2}\end{array}\right)\right)=\left(p, A_{t}\left(\begin{array}{l}z_{1} \\ z_{2}\end{array}\right)\right)$.

Realize that $A_{t}$ is contractible in $\mathrm{U}(2)$ since $\operatorname{det}\left(A_{t}\right)=1$ and $\mathrm{SU}(2)$ is simply connected. Therefore, there exists a family of loops $\tilde{A}_{t, s} \in \mathrm{U}(2)$ with $s \in[0,1]$ such that

$$
\begin{aligned}
& \tilde{A}_{t, 0}=I d \\
& \tilde{A}_{t, 1}=A_{t} .
\end{aligned}
$$

Hence, $\phi_{t, s}\left(p,\left(\begin{array}{c}z_{1} \\ z_{2}\end{array}\right)\right)=\left(p, \tilde{A}_{t, s}\left(\begin{array}{c}z_{1} \\ z_{2}\end{array}\right)\right)$ is the contraction of the positive loop.

Proof of Corollary 5. We mimic the proof of Theorem 2. A neighborhood $U_{L}$ of $L$ is diffeomorphic to $N \times \mathbb{R}^{4}$. By an application of classical $h$-principles, we can find an isotopy $\phi_{t}: N \times \mathbb{D}_{\varepsilon}^{4} \rightarrow U_{L}$ such that is the identity for $t=0$ and is a contact embedding for $t=1$.

Denote by $\varphi_{0}: L \rightarrow U_{L}$ the given Legendrian embedding. We create a path of formal Legendrian embeddings $\left(\varphi_{t}, \Phi_{t}\right)$ starting at $\varphi_{0}$ an such that $\varphi_{1}(L) \subset \phi_{1}\left(N^{+}\right) \subset$ $\phi_{1}\left(N \times \mathbb{D}_{\varepsilon}^{4}\right)$. Finally, applying twice Theorem 26 and Theorem 4 , we conclude the result.

Proof of Corollary 6. $\left(\mathbb{R}^{2 n+1}, \xi_{\text {std }}\right)$ is contactomorphic to $\left(\mathbb{R}^{2 n-3} \times \mathbb{R}^{4}\right.$, $\left.\operatorname{ker}\left(\alpha_{s t d}+r_{1}^{2} d \theta_{1}+r_{2}^{2} d \theta_{2}\right)\right)$. By compactness of the Legendrian submanifold, we can assume that $L \subset \mathbb{R}^{2 n-3} \times \mathbb{D}_{R}^{2} \times \mathbb{D}_{R}^{2}$, for some $R>0$.

Applying Lemma 23 to $N=\mathbb{R}^{2 n-3} \times \mathbb{D}_{R}^{2}(0,0)$, the domains $\mathbb{R}^{2 n-3} \times \mathbb{D}_{R}^{2}(0,0) \times$ $\mathbb{D}_{R}^{2}(0,0)$ and $\mathbb{R}^{2 n-3} \times \mathbb{D}_{R}^{2}(0,0) \times \mathbb{D}_{R}^{2}(10 R, 0)$ are contact isotopic. Therefore, we can assume that the Legendrian embedding can be pushed into $\mathbb{R}^{2 n-3} \times \mathbb{D}_{R}^{2}(0,0) \times$ $\mathbb{D}_{R}^{2}(10 R, 0) \subset\left(\mathbb{R}^{2 n-3}\right)^{+}$. We apply Theorem 4 to conclude the result.

Proof of Corollary \%. Consider $\left(\mathbb{R}^{2 n-3}, \widetilde{\xi_{o t}}=\operatorname{ker}\left(\widetilde{\alpha_{o t}}\right)\right)$ with $\widetilde{\xi_{\text {ot }}}$ any overtwisted contact structure on $\mathbb{R}^{2 n-3}$. $\left(\mathbb{R}^{2 n-3} \times \mathbb{R}^{4}, \operatorname{ker}\left(\widetilde{\alpha_{o t}}+r_{1}^{2} d \theta_{1}+r_{2}^{2} d \theta_{2}\right)\right)$ is the overtwisted at infinity contact structure on $\mathbb{R}^{2 n+1}$. The complementary of $L$ is overtwisted, thus $L$ is loose. The result follows immediately from Corollary 5 . 


\section{References}

[AFM] Albers, P., Fuchs, U. and Merry, W. J., Orderability and the Weinstein conjecture, Compos. Math. 151 (2015), 2251-2272.

[Bu] Bhupal, M., A partial order on the group of contactomorphisms of $\mathbb{R}^{2 n+1}$ via generating functions, Turkish J. Math. 25 (2001), 125-135.

[BEM] Borman, M. S., Eliashberg, Y. and Murphy, E., Existence and classification of overtwisted contact structures in all dimensions, Acta Math. 215 (2015), 281-361.

[CP] Casals, R. and Presas, F., On the strong orderability of overtwisted 3-folds, Comment. Math. Helv. 91 (2016), 305-316.

[CMP] Casals, R., Murphy, E. and Presas, F., Geometric criteria for overtwistedness. arXiv: 1503.06221.

[CPS] Casals, R., Presas, F. and Sandon, S., On the non-existence of small positive loops of contactomorphisms on overtwisted contact manifolds, J. Symplectic Geom. 14 (2016), 1013-1031.

[CN1] Chernov, V. and Nemirovski, S., Legendrian links, causality, and the low conjecture, Geom. Funct. Anal. 19 (2010), 1320-1333.

[CN2] Chernov, V. and Nemirovski, S., Non-negative Legendrian isotopy in $S\left(T^{*} M\right)$, Geom. Topol. 14 (2010), 611-626.

[CN3] Chernov, V. and Nemirovski, S., Universal orderability of Legendrian isotopy classes, J. Symplectic Geom. 14 (2016), 149-170.

[CFP] Colin, V., Ferrand, E. and Pushkar, P., Positive isotopies of Legendrian submanifolds, Int. Math. Res. Not. IMRN 2017 (2017), 6231-6254.

[CS] Colin, V. and SAndon, S., The discriminant and oscillation lengths for contact and Legendrian isotopies, J. Eur. Math. Soc. (JEMS) 17 (2015), 16571685.

[El1] EliashberG, Y., Classification of overtwisted contact structures on 3-manifolds, Invent. Math. 98 (1989), 623-637.

[El2] Eliashberg, Y., Classification of contact structures on $\mathbb{R}^{3}$, Int. Math. Res. Not. 1993 (1993), 87-91.

[EF] Eliashberg, Y. and Fraser, M., Topologically trivial Legendrian knots, J. Symplectic Geom. 7 (2009), 77-127.

[EKP] Eliashberg, Y., Kim, S. S. and Polterovich, L., Geometry of contact transformations and domains: orderability versus squeezing, Geom. Topol. 10 (2006), 1635-1747.

[EM] Eliashberg, Y. and Mishachev, N., Introduction to the h-principle, Graduate Studies in Mathematics 48, American Mathematical Society, Providence, RI, 2002.

[EP] Eliashberg, Y. and Polterovich, L., Partially ordered groups and geometry of contact transformations, Geom. Funct. Anal. 10 (2000), 1448-1476.

[Ge] Geiges, H., An Introduction to Contact Topology, Cambridge Studies in Advanced Mathematics 109, Cambridge University Press, Cambridge, 2008.

[Gi] Giroux, E., Sùr la géométrie et la dynamique des transformations de contact, Séminaire Bourbaki 1004 (2009).

[Li1] LiU, G., Positive Legendrian isotopies for loose Legendrian submanifolds, PhD 
thesis.

[Li2] LiU, G., On positive loops of loose Legendrian embeddings. arXiv : 1605. 07494.

[Mu] Murphy, E., Loose Legendrian Embeddings in High Dimensional Contact Manifolds. arXiv : 1201.2245.

[We] Weigel, P., Orderable Contact Structures on Liouville-fillable Contact Manifolds, J. Symplectic Geom. 13 (2013).

Dishant Pancholi

Institute for Mathematical Sciences

IV Cross Road, CIT Campus, Taramani

Chennai 600113, TN

India

dishant@imsc.res.in

José Luis Pérez

Instituto de Ciencias Matemáticas CSIC-

UAM-UC3M-UCM

C. Nicolás Cabrera, 13-15

Madrid 28049

Spain

joseluis.perez@icmat.es

Received October 4, 2017

in revised form January 23, 2018
Francisco Presas

Instituto de Ciencias Matemáticas CSICUAM-UC3M-UCM

C. Nicolás Cabrera, 13-15

Madrid 28049

Spain

fpresas@icmat.es 